\title{
Multicarrier Communication Techniques for Spectrum Sensing and Communication in Cognitive Radios
}

Behrouz Farhang-Boroujeny and Roland Kempter

ECE Department, University of Utah, USA

\begin{abstract}
In this tutorial article, we review different multicarrier communication methods for cognitive radio (CR) systems. There, the secondary users (SUs) need to $(a)$ dynamically and reliably determine spectral holes and (b) transmit data in these resources without interfering with other parts of the frequency band. To satisfy $(a)$, each SU has to be equipped with a spectrum analyzer. To satisfy $(b)$, it is widely accepted that a multicarrier modulation technique should be adopted. Moreover, to maximize efficiency, it has been recognized that the side-lobes of each subcarrier band must be minimized. Much of the attention in the present literature emphasizes on the use of conventional orthogonal frequency division multiplexing $(\mathrm{OFDM})$ - exploiting the fact that the fast Fourier transform (FFT) as part of the OFDM modulator can also be used for channel sensing. Herein, we discuss the performance of OFDM and also introduce filterbanks for multicarrier communication and spectral analysis in a CR setting. Moreover, the multitaper method (MTM) has been proposed as an effective method for spectrum analysis. Our article provides an insight into the pros and cons of these technologies.
\end{abstract}

\section{INTRODUCTION}

The demand for ubiquitous wireless services has been on the rise in the past and is expected to remain the same in the future. Unfortunately, the vast majority of the available spectral resources have already been licensed. It thus appears that there is little or no room to add any new services, unless some of the existing licenses are discontinued. On the other hand, studies have shown that vast portions of the licensed spectra are rarely used [12]. This has initiated the idea of cognitive radio (CR), where secondary (i.e., unlicensed) users are allowed to transmit and receive data over portions of spectra when primary (i.e., licensed) users are inactive; demanding that the secondary users (SUs) be invisible to the primary users (PUs).

To fulfill the invisibility requirement, SUs need to sense the spectrum, and this involves some sort of spectral analysis. In cases when the nature of the licensed signal is known, such analysis can be performed through feature (e.g., pilot ) detection. This is the case in IEEE 802.22 which is currently being designed to operate in the TV bands [7]. However, in the general case where such knowledge is unavailable, spectral analysis has to rely on energy detection, with likely higher requirements for sensitivity and frequency 
resolution.

\section{Motivation}

Recently, orthogonal frequency division multiplexing (OFDM) has been suggested as a candidate for multicarrier-based CR systems [2], harnessing the fact that the fast Fourier transform (FFT) as part of the OFDM demodulator can at the same time be used for spectral analysis. However, a number of shortcomings of OFDM in the application of CR have been noted. The shortcomings originate from the large side-lobes of the frequency response of the filters that characterize the subcarrier channels. These side-lobes, in turn, result in significant interference among subcarriers which originate from different SUs and between PUs and SUs. Interestingly, as of Spring 2006, the working document of the IEEE 802.22 standard covered both, frequency division duplex (FDD) as well as time division duplex (TDD) operation. In recent meetings of the standardization group, the standard has been modified to include TDD operation only. While this decision is likely inspired by the IEEE 802.16 MAC layer, it also suggests that FDD operation has been suspended because of the leakage issues of OFDM. In fact, Philips and France Telecom as the group members proposed the use of a filterbank multicarrier technique, termed offset quadrature amplitude modulation (OQAM) [5], to ease the leakage problem.

Returning to the issue of spectrum sensing, in order to reliably detect the available spectrum holes, the channel sensing mechanism needs to feature a high spectral dynamic range (SDR). If this is not the case, SUs may either interfere with low-power PUs or not be able to best harness the wireless resources. Furthermore, in order to increase the bandwidth efficiency, receivers need to have acceptable out-ofband rejection capabilities [12]. Unfortunately, as we will outline, the FFT as part of an OFDM data transmission system is neither able to provide a sufficiently high SDR for channel sensing, nor can it suit the FCC's envisioned out-of-band rejection requirements.

Other candidates for multicarrier communications in CR networks are filterbank-based systems [1]. While filterbank multicarrier communication seems to be less familiar to the cognitive radio community, it has received considerable attention from researchers in both the communications and signal processing area. Over the past three decades, three classes of such systems have been introduced. Saltzberg [4] was the first to propose a filterbank multicarrier communication system using a special quadrature amplitude modulation (QAM) technique. Prior research that initiated this development was performed by Chang 
[3]. Developments in digital subscriber line (DSL) technologies led to two other classes of filterbank multicarrier communication systems; namely, filtered multitone (FMT) [14] and discrete wavelet multitone (DWMT) modulation [13]. Out of these, DWMT has been further developed recently and renamed cosine-modulated multitone (CMT), [9]. A short overview of these methods is deferred to Section V.

We are aware that the shortcomings of OFDM/FFT in terms of leakage and reduced sensitivity may not matter much in today's CR systems. For example, the FCC requires for IEEE 802.22 to maintain large guard-bands to adjacent TV channels [7]. Also, the standard does not include frequency division multiple access (FDMA) operation, where out of the entirety of the CR "band", different clusters of subcarriers may be assigned to different users. However, as soon as true multi-user multicarrier FDMA/FDD operation is adopted, the limitations of OFDM/FFT mentioned above are likely to turn out to be significant.

As a consequence, we believe it is worthwhile to investigate the pros and cons of OFDM and filterbank multicarrier in the $\mathrm{CR}$ setting. In the following, we show that while OFDM appears as the natural candidate for multicarrier communications, it leads to inefficient spectrum utilization because of severe spectral leakage. Opposed to this, filterbank multicarrier can overcome the spectral leakage problems of OFDM and therefore lead to higher spectral efficiency. Regarding spectral sensing, we demonstrate that the FFT as part of the OFDM demodulator provides low dynamic range and thus, CRs cannot detect low power users. As the channel sensing tool, we present a novel filterbank-based spectrum analyzer as an alternative to the near-optimum Thomson's multitaper method (MTM). We demonstrate that compared to the MTM, the filterbank spectrum analyzer achieves almost identical performance at a much reduced complexity. In addition, when filterbanks are already used for data communication, filterbank channel sensing comes at virtually no additional computational cost.

\section{The Noise Floor in CR Networks}

The concept of the noise floor is a means for evaluating the effective background noise in heavily utilized parts of the spectrum. Along these lines, interference from SU sources may raise the noise floor for PUs. To quantify the effect of such sources on the primary users, the FCC spectrum policy task force has recommended the interference temperature as a new performance metric [12], [6]. Essentially, if different users' channels are at some proximity, spectral leakage among the users is likely. This will 
increase the background noise which, in turn, is equivalent to operating the system in an environment with higher temperature. Traditionally, in legacy systems, by imposing frequency masks as part of the system requirement as well as demanding sufficient separation among different user channels, leakage effects and therefore the interference temperature are minimized.

Mathematically, the interference temperature in a desired channel of width $\Delta f_{i}=f_{i+}-f_{i-}$, where $f_{i-}$ and $f_{i+}$ are the channel band edges, can be evaluated as follows. In the absence of interference from other channels/users (and ignoring electronic noise sources in the devices), the variance of the noise picked up in a bandwidth of $\Delta f_{i}$ may be calculated using the formula $\sigma_{\mathrm{n}}^{2}=k_{B} T \Delta f_{i}$, where $k_{B}=1.38 \cdot 10^{-23} \mathrm{~J} / \mathrm{K}$ denotes the Boltzmann constant and $T$ is the system temperature in Kelvin. When the leakage from other users is included, the variance of the interference picked up in the same band will be obtained as

$$
\sigma_{\text {inter }}^{2}=\sum_{j} \int_{f_{i-}}^{f_{i+}} P_{j}\left|H_{i}(f) G_{j}(f)\right|^{2} d f
$$

where $H_{i}(f)$ is the frequency response of the receiver filter of the desired channel, $P_{j}$ is the power of the $j$ th interferer at the transmitter, $G_{j}(f)$ is the channel response between the $j$ th interferer and the desired channel, and $j$ includes all the user signals that have significant contribution. Clearly, the noise and interference variances add up. This leads to the total variance $\sigma_{\text {total }}^{2}=\sigma_{\mathrm{n}}^{2}+\sigma_{\text {inter }}^{2}$, and the equivalent temperature $T_{\text {total }}=\sigma_{\text {total }}^{2} / k_{B} \Delta f_{i}$

\section{A. Reducing the interference temperature $T_{\text {total }}$}

In $\sigma_{\text {total }}^{2}=\sigma_{\mathrm{n}}^{2}+\sigma_{\text {inter }}^{2}, \sigma_{\mathrm{n}}^{2}$ depends only on the temperature of the environment and thus cannot be reduced by means of system design. From (1), one obvious way to reduce $\sigma_{\text {inter }}^{2}$ is to reduce the output power of the secondary users. However, this may not be practical. For instance, consider the situation where a secondary user which is close to a primary user, connects to a remote SU base-station. In such cases, the output power of the SU must remain high, simply to maintain connectivity. Moreover, to optimally realize the spectrum opportunities in CR scenarios with high differential power between PUs and SUs, a spectral sensor with a very wide dynamic range is a necessity.

On the other hand, $\sigma_{\text {inter }}^{2}$ is also determined by $H_{i}(f)$ and $G_{j}(f)$ and thus may be reduced by a judicious selection of these responses. In particular, (1) suggests that to minimize the interference temperature, $\left|H_{i}(f) G_{j}(f)\right|$ should be small for any $j \neq i$. In the following sections, we will show that in a filterbank multicarrier system, $\left|H_{i}(f) G_{j}(f)\right|$ can be made arbitrarily small with relatively low computational cost 
and at virtually no cost of additional bandwidth. In contrast to this, in an OFDM system, the reduction of $\left|H_{i}(f) G_{j}(f)\right|$ can be very expensive, from both a complexity and bandwidth efficiency perspective.

\section{Limitations of OFDM in Cognitive Radio Systems}

In the following, we elaborate on some shortcomings of OFDM in CR networks. In OFDM, the $i$ th subcarrier signal is characterized by a power spectral density (PSD) of the form

$$
\Phi_{i}(f)=K \operatorname{sinc}^{2}\left(\left(f-f_{i}\right) T_{S}\right)
$$

where $K$ is a constant determined by the signal level, 'sinc' is the sinc function defined as $\operatorname{sinc}(x)=$ $\sin (\pi x) / \pi x, f_{i}$ is the center frequency of the subcarrier, and $T_{S}$ is the OFDM symbol duration which consists of duration of one FFT block, $T$, and the guard interval, $T_{G}$. Assuming that the symbols in different subcarriers are independent of each other, the PSD of a OFDM signal is obtained as

$$
\Phi(f)=\sum_{i} \Phi_{i}(f)
$$

where the index $i$ includes all active subcarriers.

Because of the relatively large side-lobes of the sinc pulse in (2), the out-of-band energy generated by an OFDM signal can be significant. In a CR setting, this may result in unacceptable interference to PUs. The sinc shape of the subcarrier spectra is a consequence of the abrupt transition among successive OFDM symbols. One can avoid the sinc pulse by introducing soft transitions among successive symbols through cyclic extension of each OFDM symbol from $T_{S}$ to $(1+2 \beta) T_{S}$ and windowing by a raised cosine shape of the form shown in Fig. 1. The successive OFDM symbols are then overlapped as also shown in Fig. 1. As a result, the effective duration of each OFDM symbol is increased from $T_{S}$ to $(1+\beta) T_{S}$ which constitutes a bandwidth loss of $\beta /(1+\beta)$. In [2], the various choices of $\beta$ have been examined and it is concluded that to obtain a reasonable suppression of the out-of-band energy, values of $\beta$ as large as $\beta=1$ may be needed. To demonstrate this, here, in Fig. 2 we have presented an example of the PSD for various choices of $\beta$. This clearly shows the large side-lobes of the rectangular window $(\beta=0)$ and how the side-lobes decrease in magnitude as $\beta$ increases.

One important observation from Fig. 2 is that even though the raised cosine window is very effective in reducing the side-lobes of the subcarrier spectra, the side-lobes that are close to the main lobe are still large. This point was also noted in [2] and as a further measure to resolve the problem, a subcarrier 


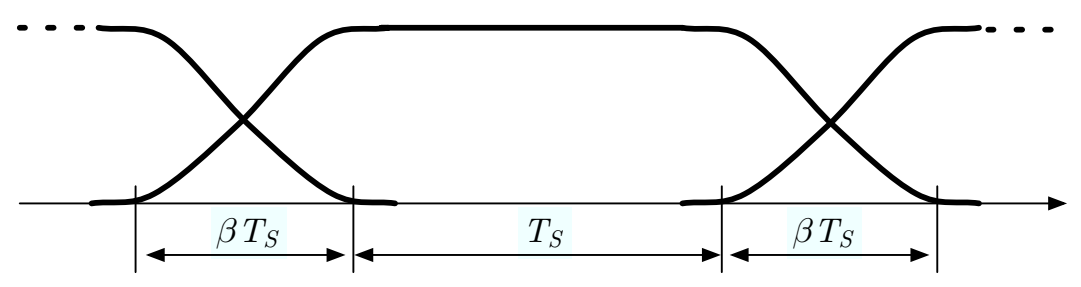

Fig. 1. Windowing at the transmitter.

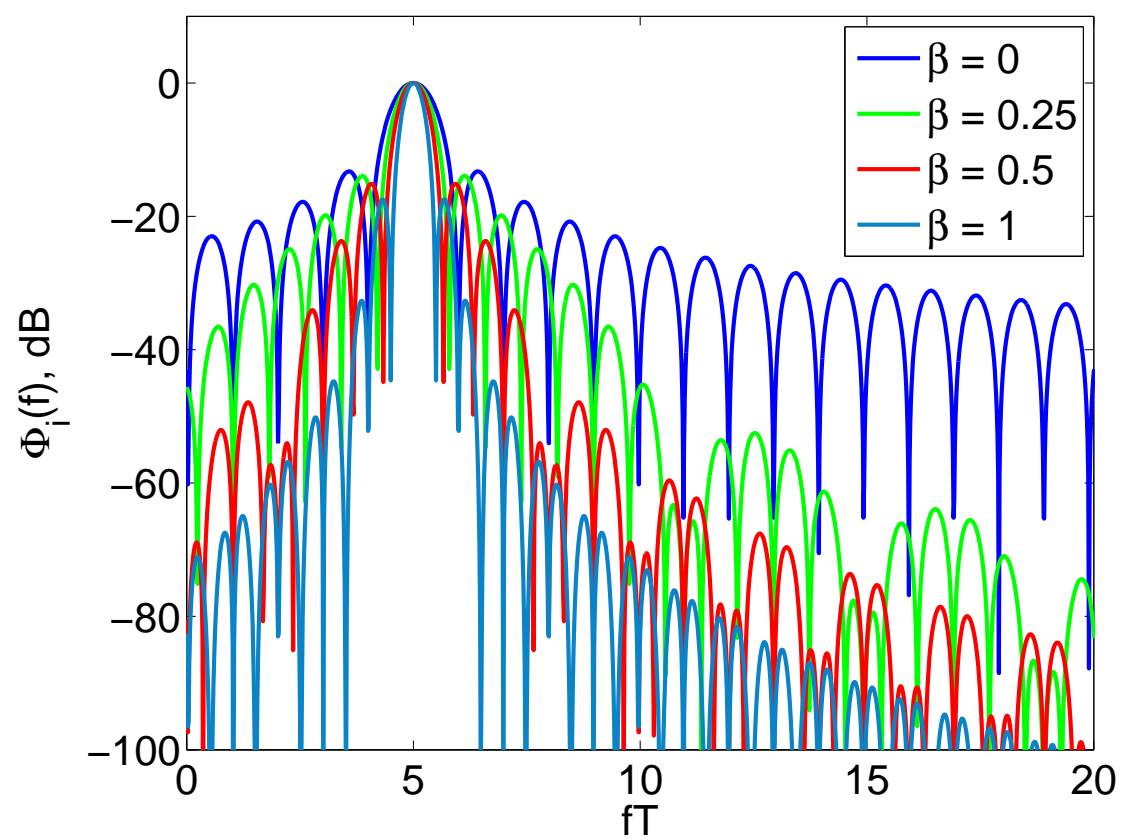

Fig. 2. The power spectral density of one subcarrier of an OFDM signal for different choices of the roll-off parameter $\beta$. Notice that the frequency axis is normalized to the subcarrier spacing $1 / T$.

deactivation mechanism that avoids subcarrier bands that are near the active PU bands was proposed. This method, clearly, further reduces the bandwidth efficiency of the CR system. In [2], it is argued that one may trade the use of smaller $\beta$ (less bandwidth loss due to raised cosine window) for a larger number of deactivated subcarriers.

Another interesting development is by Brandes et al. [11], where the authors propose a method for further reduction of the side-lobes by assigning non-zero values to the deactivated subcarriers to reduce the magnitude of the side-lobes generated by the data subcarriers. The results presented in [11] report the use of $\beta=0.2$ and can achieve side-lobes at around $-60 \mathrm{~dB}$. However, the selection of cancellation subcarriers involves a constraint optimization that must be repeated for each OFDM symbol.

Another important point that deserves some attention is the interference received by an SU from PUs and other SUs. Weiss et al. [2] acknowledged the issue without proposing any method of reducing it. We 
recall from the DSL literature [8] that this problem can be solved by applying a window to the received signal prior to passing it to the FFT block for demodulation. The concept of windowing here is somewhat similar to the one used at the transmitter. However, differences exist. Fig. 3 depicts the method of receiver windowing. When the window is rectangular, one picks $N$ samples of a received OFDM symbol, after removing the cyclic prefix samples, and passes them to an $N$-point FFT for demodulation. To apply a raised cosine window, $(1+\alpha) N$ samples are chosen and windowed as also shown in Fig. 3 . In order to obtain samples of the windowed signal in the frequency domain at the middle of the $N$ subcarrier bands, a Fourier transform has to be applied to $(1+\alpha) N$ time-domain samples, followed by decimation to $N$ output samples. This can be realized by aliasing in the time-domain and applying an $N$-point FFT. The arrows in Fig. 3 demonstrate how this is performed; essentially, the samples in the shaded areas are added to the windowed samples at the two corners of the time period $T$. Clearly, application of windowing at the receiver also requires the addition of cyclic prefix and suffix samples which will further reduce the bandwidth efficiency of OFDM.

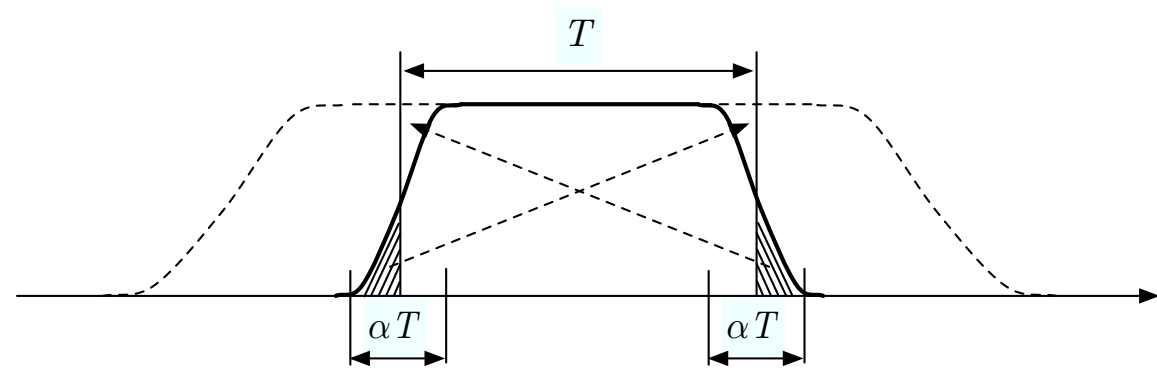

Fig. 3. Windowing at the receiver.

\section{Filterbank Multicarrier Communication Techniques}

Pioneering work on filterbank multicarrier communication techniques was done by Chang [3] and Saltzberg [4] in the mid 1960's. Saltzberg showed that by proper design of a transmit pulse-shape in a multichannel QAM system, and by introducing a half symbol space delay between the in-phase and quadrature components of QAM symbols, it is possible to achieve a baud-rate spacing between adjacent subcarrier channels and still recover the information symbol, free of intersymbol (ISI) and intercarrier interference (ICI). This leads to maximum spectral efficiency. Further progress was made by Hirosaki [5] who showed that the transmitter and receiver part of this modulation method could be implemented efficiently in a polyphase/DFT structure. The method was called orthogonally multiplexed 
QAM (OQAM) in [5]. OQAM has later been referred to as OFDM-OQAM, with the acronym OQAM standing for offset $Q A M$, reflecting the fact that the in-phase and quadrature of each QAM symbol are time offset with respect to each other.

In the 1990's, the advancements in DSL technology motivated more activity in the development of other filterbank-based multicarrier communication systems to better suit DSL channels. Early development in this area is an ANSI contribution by Tzannes et al., which was later expanded and called discrete wavelet multitone (DWMT) [13]. In [9], it was shown that DWMT uses cosine-modulated filterbanks that are more frequently used for signal compression. The name cosine modulated multitone (CMT) was later adopted for this class of modulators. Other interesting advancements that were reported in [9] are: (i) in CMT, each subcarrier channel transmits a PAM symbol using vestigial sideband (VSB) modulation, and (ii) the very particular structure of the subcarrier signals in CMT leads to a simple blind detection algorithm.

Filtered multitone (FMT) is another multicarrier modulation technique specifically developed for DSL applications [14]. As opposed to OFDM-OQAM and CMT, which allow for overlapping of adjacent subcarrier bands, in FMT subcarrier bands are disjoint. It is thus less bandwidth efficient than FMT and OFDM-OQAM.

\section{Vi. Spectrum Sensing Methods}

In non-parametric spectral estimation, an estimate of the PSD of a random process $x(n)$ is obtained by passing $x(n)$ through a bank of narrowband bandpass filters and measuring the average output power of these filters [10]. The simplest member of this class of spectral estimators is the periodogram spectral estimator (PSE), where a bank of filters whose coefficients are those of the discrete Fourier transform (DFT) are used. A simple method of increasing the SDR is to apply a window to the samples of $x(n)$ prior to application of the FFT. This method, which is known as the Blackman-Tukey spectral estimator (BTSE), is widely used in practice [10] and has been proposed in [2] for spectrum sensing.

The Multitaper method (MTM) is one of the most advanced non-parametric spectral estimation methods. In MTM, each point of the desired PSD is obtained by averaging the signal power at the output of a set of narrowband (known as prolate) filters that have the same passband, but have been designed to generate a set of independent outputs to minimize the variance of the estimates. Moreover, the MTM 
filters are designed to provide maximum attenuation in their stop-band - resulting in maximum SDR. Recently, Haykin [6] has identified MTM as the best method for channel sensing in CR, because of its near optimal performance.

In a filterbank multicarrier communication system, the receiver front end consists of a bank of narrowband filters which are used to separate different subcarrier signals. The output power of these filters may also be used to estimate the PSD of the received signal. Hence, spectral sensing can be established at virtually no additional cost. Since the analysis filters are highly tuned to subcarrier frequencies and can be laid out for any arbitrarily low stopband gain, a filterbank spectrum analyzer can be designed for any desired SDR.

For comparison of the various spectral estimation methods, we generate a random process $x(n)$ as the received signal in a $\mathrm{CR}$ and explore the outcomes of the various spectral estimation methods. The process $x(n)$ that we have selected has three passbands with signal levels that spans over a wide range of $60 \mathrm{~dB}$. This wide range allows us to explore the SDR of the various methods.

For all cases we assume that the PSD estimate is obtained from 2048 consecutive samples of $x(n)$. We also assume that the desired frequency resolution within the band of interest is 256 subbands. Thus, if the conventional OFDM is used for communication as well as for spectrum sensing, frames of length 256 samples of $x(n)$ are processed and the output power of each subcarrier is averaged over $8(=2048 / 256)$ frames. We present the results of PSE, i.e., when FFT is applied without any windowing, and when BTSE with a Hanning window is applied.

In the MTM, we examine the outcomes of using 3, 4 and 5 prolate filters of length 2048. The responses of the five prolate filters, after modulation to the center frequency $f_{i}=0.025$, are shown in Fig. 4 . We observe that only the first 3 prolate filters have a stopband of below $-60 \mathrm{~dB}$ and, thus, can support the desired SDR $60 \mathrm{~dB}$.

In the case of filterbank spectral estimation, we consider a filterbank that we have designed for demodulation in an OFDM-OQAM system with 256 subcarrier channels. The length of the prototype filter in the filterbank is 1536 . The PSD estimate is obtained by averaging the power of the three consecutive samples at the output of each subcarrier bank. This corresponds to a span of 2048 samples of $x(n)$ for a fair comparison to other methods. Magnitude responses of the prototype filter, when modulated to three adjacent bands, are shown in Fig. 5. The stopband attenuation of better than $-60 \mathrm{~dB}$ in these filters 


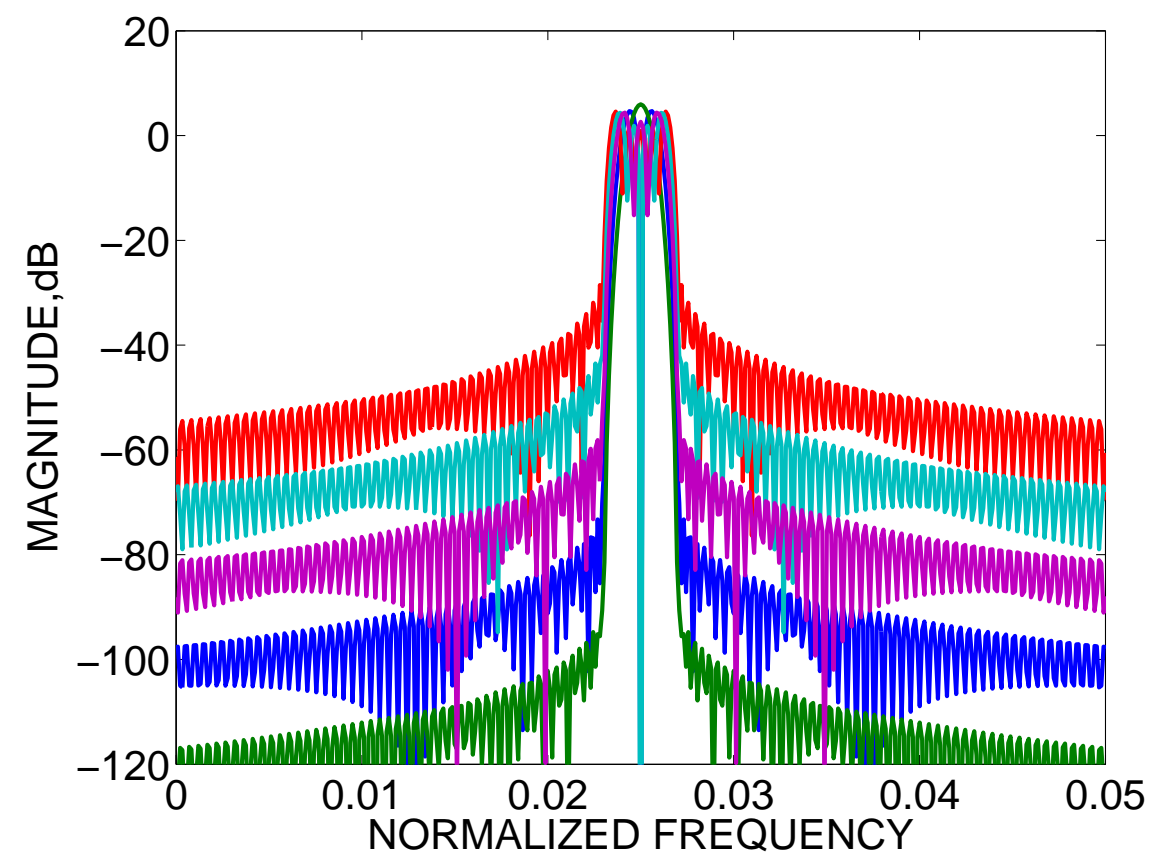

Fig. 4. Magnitude responses of five prolate filters of length 2048. Notice that only the first three prolate filters have stopband gains of below $-60 \mathrm{~dB}$.

guarantees the target SDR of $60 \mathrm{~dB}$.

Fig. 6 presents results for the various spectral estimators. The PSD that the various method strive to estimate is also shown (labeled 'exact'). From these results, we see that the MTM with three prolate filters and the filterbank method have very similar performance and easily accommodate an SDR of $60 \mathrm{~dB}$. The MTM with more than three prolate filters begins to loss its SDR, as was predicted above. PSE performs very poorly. BTSE improve the latter result, but still performs poorly near the band-edges.

\section{ViI. Conclusion and Outlook into Future Research}

We discussed the conventional OFDM and filterbank multicarrier communication and sensing techniques in CR systems. While the eminent role of OFDM in the current standards was acknowledged, we pointed out that OFDM suffers from leakage issues which may considerably limit its usability in future CR networks. We noted that, just like OFDM, filterbanks can be used as an integrated tool for data communication as well as channel sensing. We demonstrated that when used as a data communication technique, filterbank multicarrier modulation offers much higher spectral efficiency than OFDM. Furthermore, when used for spectrum analysis, compared to the optimal multitaper method (MTM), filterbanks can be nearly as powerful, while offering greatly reduced computational complexity. In addition, since filterbanks offer full control over the spectral leakage, they allow to dynamically adjust the interference 


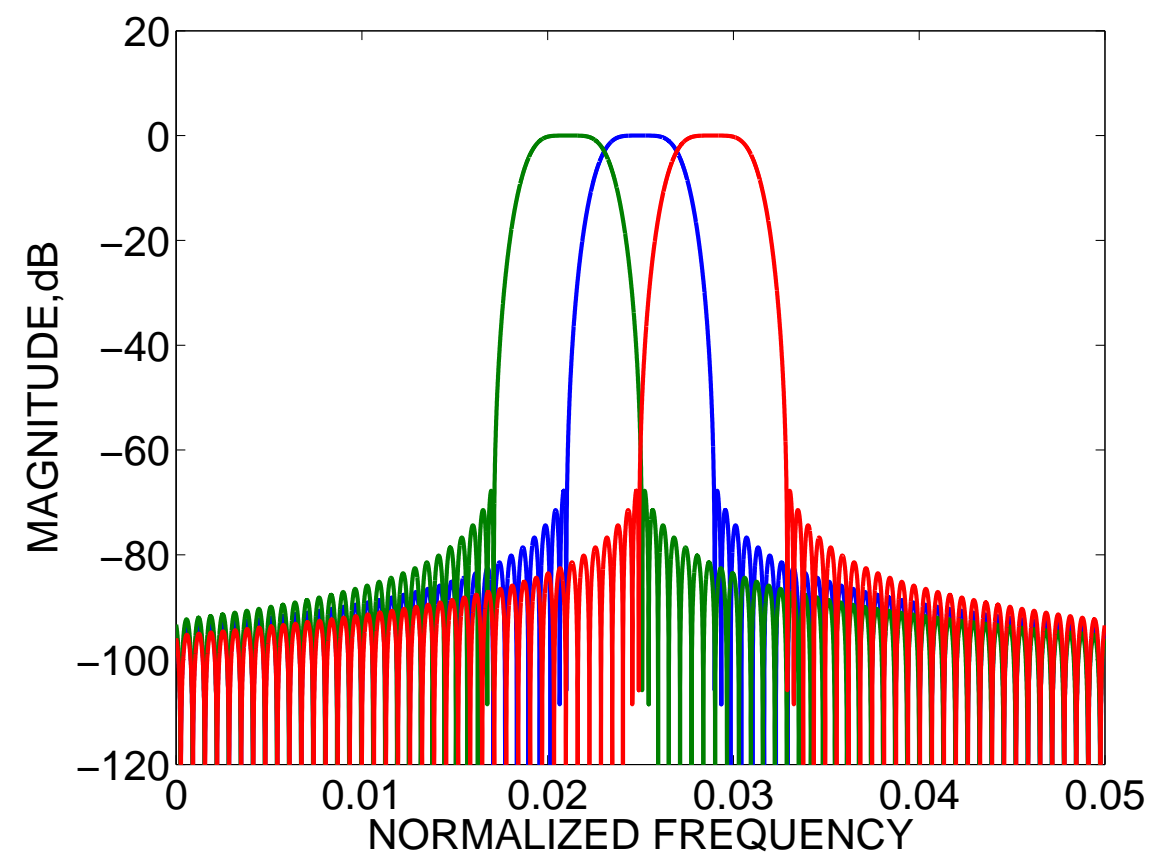

Fig. 5. Magnitude responses of three adjacent subcarrier filters in a 256 channel OFDM-OQAM system. Filter length is $256 \times 6=1536$. Notice that a stopband gain of less than $-60 \mathrm{~dB}$ is acheived.

temperature - a desirable goal suggested by the FCC for CR systems. As a result, filterbank multicarrier modulation is a natural candidate for spectrum sensing and data communication in cognitive radio systems. However, unlike conventional OFDM, which has been extensively studied and is well understood, filterbank multicarrier methods have so far received very limited attention. Hence, one of the goals of this magazine article was to disseminate the basic knowledge of filterbank multicarrier techniques to the community of communication and signal processing engineers and, hopefully, to initiate a wider study of this important signal processing tool.

\section{REFERENCES}

[1] P. Amini, R. Kempter, R. R. Chen, L. Lin and B. Farhang-Boroujeny, "Filter Bank Multitone: A Physical Layer Candidate for Cognitive Radios," Software Defined Radio Technical Conference, SDR 2005, November 14-18, 2005, Orange County/California, available at http://www.flux.utah.edu/ rolke/content/SDR05_FB_CR.pdf

[2] T.A. Weiss and F.K. Jondral, "Spectrum pooling: an innovative strategy for the enhancement of spectrum efficiency," IEEE Communications Magazine, Vol. 42, No. 3, March 2004, pp. S8 - S14.

[3] R.W. Chang, "High-speed multichannel data transmission with bandlimited orthogonal signals," Bell Sys. Tech. J., vol. 45, pp. 1775-1796, Dec. 1966.

[4] B.R. Saltzberg, "Performance of an efficient parallel data transmission system," IEEE Trans. on Comm. Tech., vol. 15, no. 6, pp. 805-811, Dec. 1967.

[5] B. Hirosaki, "An Orthogonally Multiplexed QAM System Using the Discrete Fourier Transform," IEEE Transactions on Communications, Volume 29, Issue 7, Jul 1981 Page(s): 982 - 989

[6] S. Haykin, "Cognitive radio: brain-empowered wireless communications," IEEE Journal Selected Areas in Communications, vol. 23, no. 3, pp. 201-220, Feb. 2005.

[7] The IEEE 802 LAN/MAN Standards Committee, 802.22 WG on Wireless Regional Area Networks (WRANs), http://www. ieee802.org/22/

[8] D. G. Mestdagh, M. R. Isaksson, and P. Odling, "Zipper VDSL: a solution for robust duplex communication over telephone lines," IEEE Communications Magazine, vol. 38, no. 5, pp. 90-96, May 2000. 


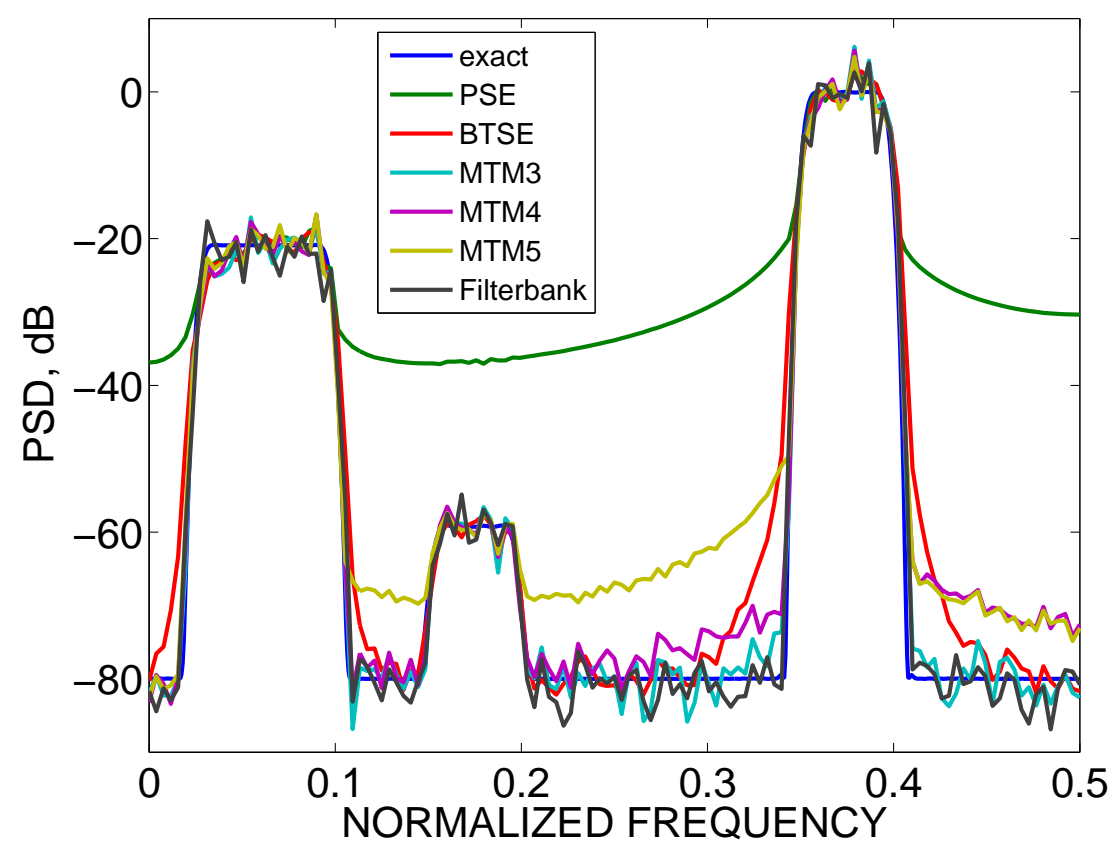

Fig. 6. An example of power spectral density (PSD) of random process, and snapshots of various spectral estimation methods.

[9] B. Farhang-Boroujeny, "Multicarrier modulation with blind detection capability using cosine modulated filter banks," IEEE Transactions on Communications, vol. 51,no. 12, pp. 2057-2070, Dec. 2003.

[10] J.S. Lim and A.V. Oppenheim (Editors), Advanced Topics in Signal Processing. Prentice Hall, Englewood Cliffs, New Jersey, 1988.

[11] S. Brandes, I. Cosovic, M. Schnell, "Reduction of Out-of-band Radiation in OFDM Based Overlay Systems," First IEEE International Symposium on New Frontiers in Dynamic Spectrum Access Networks, DySPAN 2005, 8-11 Nov. 2005 Page(s):662 - 665

[12] Federal Communications Commission, "Spectrum Policy Task Force," rep. ET Docket no. 02-135, Nov. 2002

[13] S.D. Sandberg and M.A. Tzannes, "Overlapped Discrete Multitone Modulation for High Speed Copper Wire Communications," IEEE Journal on Selected Areas in Communications, vol. 13, no. 9, pp. 1571-1585, Dec. 1995.

[14] G. Cherubini, E. Eleftheriou, S. Olcer, J.M. Cioffi, "Filter bank modulation techniques for very high speed digital subscriber lines," IEEE Communications Mag., vol. 38, no. 5, pp. 98-104, May 2000. 